\title{
A Computational Insight into Reaction Between Different Amino Acids with Reactive Aldehydes 4-hydroxy-2-nonenal and 4-oxo-2-nonenal
}

\author{
Sanja Škulj, Mario Vazdar*
}

Division of Organic Chemistry and Biochemistry, Ruđer Bošković Institute, Bijenička c. 54, HR-10000 Zagreb, Croatia

* Corresponding author's e-mail address: mario.vazdar@irb.hr

RECEIVED: July 8, 2019 * REVISED: August 28, 2019 * ACCEPTED: August 29, 2019

This Paper is Dedicated to Prof. Kata Mlinarić-Majerski ON THE OCCASION OF Her 70 $70^{\text {Th }}$ BIRTHDAY

\begin{abstract}
In this work, we studied in detail the reaction mechanism of modification of arginine (Arg), cysteine (Cys) and histidine (His) model amino acids upon the reaction with biologically relevant reactive aldehydes 4-hydroxy-2-nonenal (HNE) and 4-oxo-2-nonenal (ONE) in acetonitrile and acetonitrile/water systems by using high level ab initio calculations. We identified and characterized all of the reaction steps along two possible pathways - Michael addition pathway and Schiff base pathway resulting in the formation of Michael adducts/hemiacetals and carbinolamine/Schiff base adducts, depending on the reactive aldehyde and the reaction pathway. Overall energetics suggests that Arg amino acid is more reactive than Cys and His amino acids in both reaction pathways. We established that the ONE is in general more reactive than $\mathrm{HNE}$ and also found out that addition of water in the reaction steps involving proton transfer strongly catalyzes the reaction by decreasing prohibitively high free energy barriers.
\end{abstract}

Keywords: amino acids, 4-hydroxy-2-nonenal, 4-oxo-2-nonenal, quantum chemical calculations, oxidative stress.

\section{INTRODUCTION}

$\mathbf{R}$ EACTION between amino acids and biologically relevant reactive aldehydes (RAs), such as 4-hydroxy2-nonenal (HNE) ${ }^{[1]}$ and 4-oxo-2-nonenal (ONE) ${ }^{[2]}$ which are constantly generated during oxidative stress, ${ }^{[3]}$ represents one of the main non-enzymatic post-translational modifications of proteins and lipids in living organisms. ${ }^{[4,5]}$ In particular, amino acids, such as lysine (Lys), arginine (Arg), histidine (His) and cysteine (Cys), undergo nucleophilic addition to one of the reactive sites in HNE and ONE. ${ }^{66,7]}$ The covalent modifications of proteins induced by RAs result in various irreversible protein adducts, such as Michael and Schiff base adducts, ${ }^{[6]}$ which can alter their normal physiological function. Usually, modified proteins are formed in relatively low concentrations during oxidative stress due to low RA concentration in physiological conditions and are swiftly metabolized by the cellular proteosomal system. ${ }^{\left[{ }^{[8]}\right.}$ However, if the concentration of RAs generated during oxidative stress is very high, for example in pathological conditions, then the removal of modified proteins is not very efficient and can lead to severe consequences and diseases, such as diabetes 2 , cancer, Parkinson's disease, Alzheimer's disease or atherosclerosis. ${ }^{[8-12]}$

The covalent modifications of various amino acids have been extensively reported in the literature. Above all, it is important to highlight the work of Sayre and his group, which were one of the pioneers in the investigation of amino acid modifications induced by RAs. ${ }^{[13-15]}$ In particular, Sayre and coworkers have found that ONE is more neurotoxic and more reactive than HNE towards amino acids, and that cysteine is the most reactive amino acid in the series Cys $\gg$ His $>$ Lys $>$ Arg in water. ${ }^{[16]}$ Petersen and coworkers also identified various products between HNE and ONE with model peptides by MALDI-TOF-MS, additionally confirming previous findings. ${ }^{[17]}$ Incidentally, Petersen and coworkers have not detected that Arg amino acid makes adducts with $\mathrm{HNE}$, 


\section{Michael adduct pathway}<smiles>[R]C(/C=C/C=O)CCCCC</smiles>

1

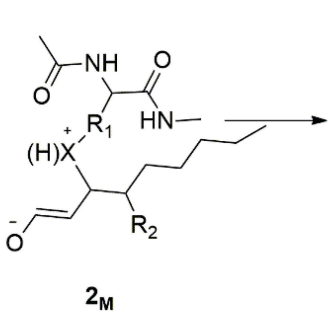<smiles>[R]C(CC=O)C(O)CCCCC</smiles><smiles>[R][C@H](NC(C)=O)C(=O)NC[13CH3]</smiles>

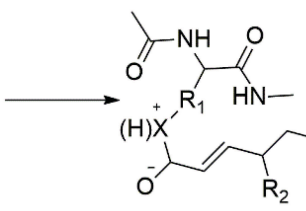

$2 \mathrm{~s}$<smiles>[Y]C(O)/C=C/C([R2])CCCCC</smiles>

3s<smiles>[R]C(=CC=CC([R])CCCCC)C(NC(C)=O)C(=O)NC</smiles>

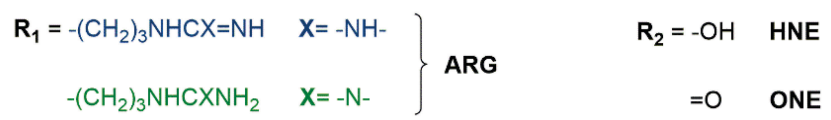

$$
\begin{aligned}
& -\mathrm{CH}_{2} \mathrm{X} \quad \mathrm{X}=-\mathrm{S}-\quad \text { CYS } \\
& \text { " } X{ }^{\prime \prime} \quad X=-\mathrm{N}-\text { HIS }
\end{aligned}
$$

Figure 1. General reaction mechanism between amino acid (arginine, cysteine, histidine) with HNE and ONE, respectively, resulting in various Michael adducts and Schiff base adducts.

only with ONE. In addition to amino acids and small peptides, there have been numerous studies in the past on the addition of HNE and ONE to proteins, such as oxidoreductases, hydrolases, ion channels and cytoskeletal proteins. ${ }^{[5,18,19]}$ In many cases, the most frequently modified amino acid is cysteine due to its presumably lowest reactivity, but modifications of histidine, lysine and arginine have also been found.

In our previous work, we have revisited in detail the reaction mechanism between model lysine amino acid and HNE in organic solvents containing different amounts of water. ${ }^{[20]}$ We have shown a plethora of different reaction routes and products dependent on the water content, which have been identified by different spectroscopic techniques such as NMR and LC-MS. In particular, we have shown that the amino group readily reacts with either the $\mathrm{C}=\mathrm{C}$ double bond in HNE or its carbonyl group, resulting in Michael and Schiff adducts as well as subsequent pyrrole and pyrimidinium salt adducts. Moreover, we have found out that increasing water content yields more polar products, such as Michael adduct and pyrimidinium salt adduct, in contrast to nonpolar solvents where nonpolar pyrrole adduct has been detected as a major product.

However, as other amino acids can also react with RAs as exemplified in the previous paragraphs, we will focus here on the reaction mechanism of other amino acids Cys, His and Arg with HNE and ONE, respectively, with the aim of elucidating and quantifying the reaction mechanism energetics which will be compared to Lys amino acid reactivity using identical computational setup as in the previous work. An overview of the general reaction mechanism between different amino acids and HNE and ONE, respectively, inspired by our previous studies and experimental evidence is presented in Figure 1. To the best of our knowledge, no computational studies aiming to clarify the reaction mechanism between RAs and Arg, Cys and His have been reported before, and this will represent the main topic of this work.

\section{Computational Methods}

Quantum chemical calculations of reaction mechanism of reaction between model $\mathrm{Arg}$, His and Cys side chain 
(protected by acetyl and N-methyl groups) and HNE and ONE (Figure 1), respectively, were performed in neat polar aprotic solvent acetonitrile $(\varepsilon=35.7)$ (ACN setup) and acetonitrile with one explicit water molecule ( $\mathrm{ACN}+\mathrm{W}$ setup) using SMD solvation model[21] at $298 \mathrm{~K}$ and 1 bar, similar to our previous work. ${ }^{[20]}$ Despite limitations of polarizable continuum models (such as incapacity for appropriate accounting of entropy effects and thermal averaging), the use of implicit solvent models represents a good compromise for the systems of interest. ${ }^{[22]}$ All calculations were obtained using SMD/MP2/6$311++G(d, p) / / B 3 L Y P / 6-31 G(d)$ level of theory, i.e. geometry optimizations were calculated at the SMD/B3LYP/6$31 \mathrm{G}(\mathrm{d})$ level of theory[ ${ }^{[23,24]}$ followed by MP2 single-point calculations on the optimized structures at the SMD/MP2/6-311++G(d,p) level of theory. ${ }^{[25,26]}$ Stationary points, minima and transition states on the potential energy surface were identified by vibrational analysis. Transition state structures were verified by the presence of one negative eigenvalue, and by inspecting the displacement along the vibrational mode corresponding to the imaginary frequency. Gibbs free energies were calculated as a sum of single-point electronic energy and thermal correction to Gibbs free energy. All quantum chemical computations have been performed using Gaussian09 suite of codes.[27]

\section{RESULTS AND DISCUSSION}

Figure 1 shows an overview of a general reaction mechanism valid for the reaction mechanism between studied amino acids and RAs. We see that two possible pathways are responsible for two different product classes, namely Michael adduct pathway (1,4-nucleophilic addition) and Schiff base adduct pathway (1,2-nucleophilic addition). In the Michael adduct pathway, amino acid of interest reacts either with HNE and ONE, first resulting in the (possible) formation of a zwitterionic intermediate by nucleophilic addition of amino acid to the double bond of RA, which subsequently transforms to a Michael adduct by proton transfer reaction. After formation of a Michael adduct, in the case of HNE (but not ONE) an additional cyclization step involving proton transfer yields a hemiacetal adduct, which is the final product located in this pathway. On the other hand, a first step of the reaction in the Schiff base pathway is nucleophilic addition to the carbonyl group of RA, forming again a zwitterionic intermediate which can subsequently transform by proton transfer reactions and carbon-carbon coupling to a carbinolamine (or hemithioacetal adduct in the case of Cys) and a Schiff base adduct after elimination of water, respectively. In contrast to reaction of lysine with $\mathrm{HNE}$, where the reaction can continue and finish in more complicated pyrrole and pyrimidinium salt adduct by further cyclization of a Schiff base adduct, ${ }^{[20]}$ in the cases of Cys and His the formation of a carbinolamine (or hemithioacetal in the case of Cys) adduct with both HNE and ONE is actually the final product, whereas for the reaction of Arg with HNE and ONE the final product is a Schiff base adduct. In the following paragraphs, we will examine the reaction mechanism of reaction between Arg, Cys and His with HNE and ONE, respectively, and we will describe all the details of the reaction mechanism and its energetics.

\section{Modification of Amino Acids with HNE}

In this Section, we will briefly give an overview of possible reaction products between $\mathrm{Arg}$, Cys and His amino acids with $\mathrm{HNE}$, showing possible reaction routes leading to different products depending on the reaction pathway. After examination of possible reaction routes, we will study the energetics of the particular reaction in detail.

As a first example, in the case of reaction between arginine and HNE, two nucleophilic sites of Arg can be involved in reaction with double bond in HNE (Figure 2) both resulting in Michael adduct and hemiacetal adducts as reaction products (Figure 1). The difference in the reaction is in the nucleophilic group of Arg amino acid which in its neutral form possesses two possible nucleophilic sites located in the side chain guanidinium group, $-\mathrm{NH}_{2}$ (Figure $2 \mathrm{a}$ ) and $=\mathrm{NH}$ (Figure $2 \mathrm{~b}$ ), which can both react with HNE. In both cases, the reaction leads to Michael and hemiacetal adducts which only differ in the adduction site of Arg.

According to the Schiff base adduct pathway, the addition of Arg also proceeds via two nucleophilic sites

Michael adduct pathway

a)
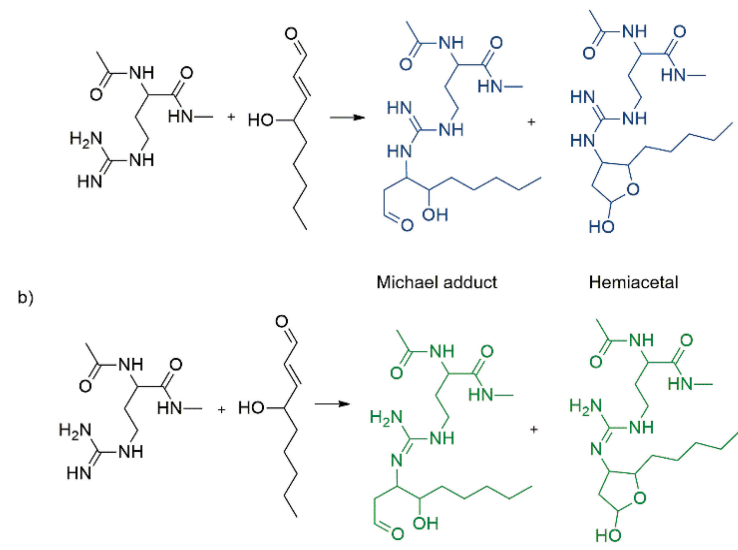

Michael adduct

Hemiacetal

Figure 2. Michael adduct pathway for reaction between $\mathrm{NH}_{2}$ group of arginine (panel a) and $=\mathrm{NH}$ group of arginine (panel b) with HNE. 
(Figure 3) - however, when Arg reacts with its $-\mathrm{NH}_{2}$ group, the reaction can proceed to the final Schiff base adduct carbinolamine intermediates and subsequent water elimination (Figure 3a), whereas it stops in the carbinolamine intermediate when $=\mathrm{NH}$ group reacts with HNE (Figure $3 b$ ) since no protons are available for the subsequent proton transfer and elimination of water.

In the case of Cys, there is only one nucleophilic site (-SH group), which can react with HNE. The Michael adduct pathway leads to Michael and hemiacetal adducts (Figure 4a), whereas the Schiff base adduct pathway leads only to hemithioacetal adduct and Schiff base cannot be formed (Figure 4b).

Finally, in the case of His modification with HNE, the situation is similar to Cys amino acid, where we found that $-\mathrm{NH}$ group in imidazole side chain ring reacts with HNE along the Michael adduct pathway resulting in both Michael and hemiacetal adducts (Figure 5a) and only the carbinolamine adduct in the case of the Schiff base adduct pathway (Figure $5 b$ ).

Now we turn to the energetics of the reaction. Table 1 shows calculated free energies of the reaction at the SMD/MP2/6-311++G(d,p)//B3LYP/6-31G(d) level of theory in two different setups, $\mathrm{ACN}$ and $\mathrm{ACN}+\mathrm{W}$ (see Computational Details). Free energy diagram showing reaction mechanism for formation of described adducts for all

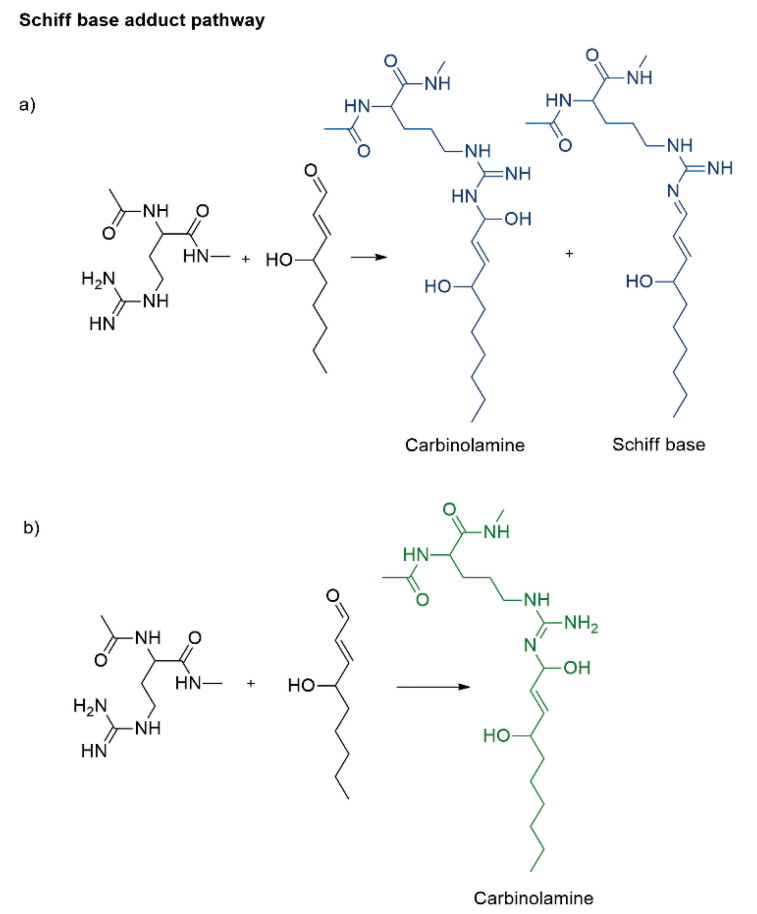

Figure 3. Schiff base adduct pathway for reaction between $-\mathrm{NH}_{2}$ group of arginine (panel a) and $=\mathrm{NH}$ group of arginine (panel b) with HNE. amino acids after reaction with HNE in acetonitrile (ACN setup) is presented in Figure 6, whereas free energy diagram for the reaction mechanism with one explicit water in acetonitrile ( $\mathrm{ACN}+\mathrm{W}$ setup) is presented in Figure 7.

Michael adduct pathway

a)

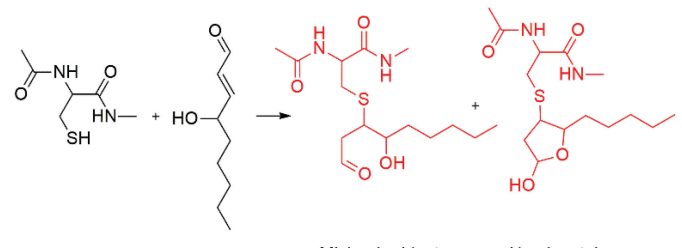

Michael adduct Hemiacetal

Schiff base adduct pathway

b)

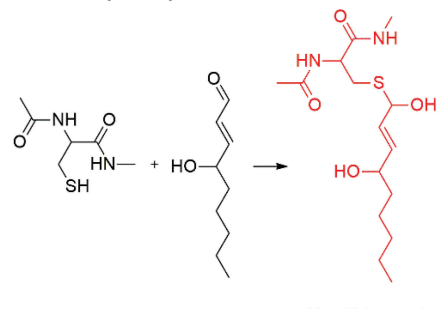

Figure 4. Michael adduct pathway (panel a) and Schiff base adduct pathway (panel b) for reaction between $-\mathrm{SH}$ group of cysteine and HNE.

Michael adduct pathway

a)<smiles>CCCCCCC(O)CCCCC</smiles>

Michael adduct Hemiacetal

Schiff base adduct pathway

b)

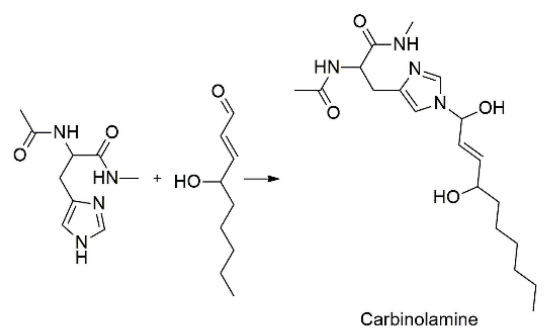

Figure 5. Michael adduct pathway (panel a) and Schiff base adduct pathway (panel b) for reaction between $-\mathrm{NH}$ group of histidine and HNE. 
Table 1. Relative free energies of studied compounds vs. reactants in $\mathrm{kcal} \mathrm{mol}^{-1}$ for the reaction between HNE and different amino acids along Michael adduct pathway (MA pathway) and Schiff base adduct pathway (SB pathway) calculated at the $\mathrm{SMD} / \mathrm{MP} 2 / 6-311++\mathrm{G}(\mathrm{d}, \mathrm{p}) / / \mathrm{B} 3 \mathrm{LYP} / 6-31 \mathrm{G}(\mathrm{d})$ level of theory in acetonitrile (ACN setup) and acetonitrile with one explicit water molecule (ACN+W setup).

\begin{tabular}{|c|c|c|c|c|c|c|c|c|c|c|}
\hline \multirow{2}{*}{ HNE } & \multicolumn{5}{|c|}{ ACN setup } & \multicolumn{5}{|c|}{$A C N+W$ setup } \\
\hline & $\mathrm{Arg}-\mathrm{NH}_{2}$ & $\mathrm{Arg}=\mathrm{NH}$ & Cys - SH & $\mathrm{His}-\mathrm{NH}$ & Lys ${ }^{(a)}-\mathrm{NH}_{2}$ & $\mathrm{Arg}-\mathrm{NH}_{2}$ & $\mathrm{Arg}=\mathrm{NH}$ & Cys - SH & $\mathrm{His}-\mathrm{NH}$ & Lys $^{(a)}-\mathrm{NH}_{2}$ \\
\hline \multicolumn{11}{|c|}{ MA-pathway } \\
\hline 1 & 0.0 & 0.0 & 0.0 & 0.0 & 0.0 & 0.0 & 0.0 & 0.0 & 0.0 & 0.0 \\
\hline $\mathrm{TS}_{1 \mathrm{M}}$ & - & 20.9 & - & - & 15.2 & 34.3 & 18.7 & - & - & 15.0 \\
\hline $2 M$ & - & 10.2 & - & - & 8.2 & 31.9 & 9.1 & - & - & 6.3 \\
\hline $\mathrm{TS}_{2 \mathrm{M}}$ & 44.0 & 37.7 & 55.7 & 59.6 & 32.7 & 37.7 & 22.3 & 46.2 & 53.0 & 17.9 \\
\hline $3 \mathrm{M}$ & -3.3 & -3.6 & -12.6 & -11.1 & -7.1 & -0.6 & -6.2 & -13.7 & -8.0 & -10.5 \\
\hline $\mathrm{TS}_{3 \mathrm{M}}$ & 34.4 & 33.5 & 23.8 & 26.2 & 30.9 & 21.7 & 20.9 & 7.3 & 14.6 & 12.8 \\
\hline $4 M$ & -3.8 & -5.3 & -14.0 & -11.6 & -9.1 & -1.9 & -5.8 & -15.6 & -7.7 & -10.5 \\
\hline \multicolumn{11}{|c|}{ SB-pathway } \\
\hline 1 & 0.0 & 0.0 & 0.0 & 0.0 & 0.0 & 0.0 & 0.0 & 0.0 & 0.0 & 0.0 \\
\hline $\mathrm{TS}_{1 \mathrm{~S}}$ & - & - & - & - & - & - & 17.4 & - & - & - \\
\hline $2 s$ & - & - & - & - & - & - & 13.6 & - & - & - \\
\hline $\mathrm{TS}_{2 \mathrm{~S}}$ & 43.6 & 33.7 & 40.3 & 43.7 & 27.9 & 26.8 & 15.5 & 25.3 & 34.2 & 10.0 \\
\hline $3 \mathrm{~s}$ & 5.2 & 2.6 & 0.3 & -0.6 & -0.8 & 5.6 & 3.4 & -1.5 & -1.7 & -2.9 \\
\hline $\mathrm{TS}_{3 \mathrm{~S}}$ & 54.8 & - & - & - & 39.3 & 42.5 & - & - & - & 22.9 \\
\hline 4 & 3.9 & - & - & - & -5.4 & -1.2 & - & - & - & -7.3 \\
\hline
\end{tabular}

(a) Taken from Ref. [20].

First, we will focus on the reaction mechanism between amino acids and HNE along the Michael adduct pathway in neat acetonitrile (ACN setup, Figure 6). As a first step of the reaction, an amino acid (Arg, Cys, His) undergoes nucleophilic addition towards the HNE double bond,

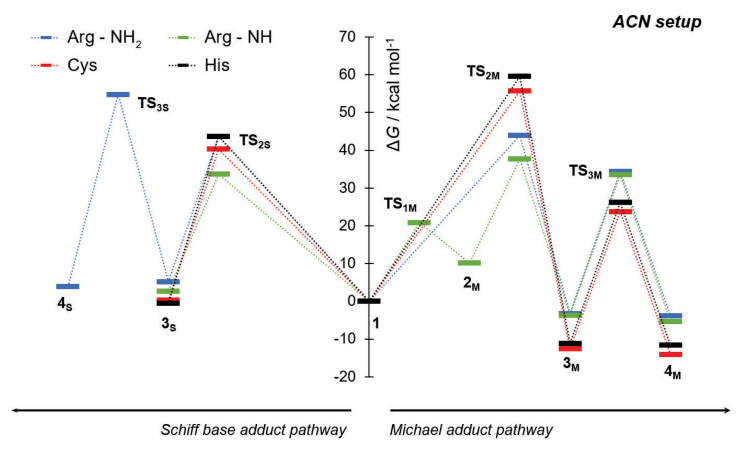

Figure 6. Free energy diagram for Michael adduct pathway (to the right) and Schiff base adduct pathway (to the left) for reaction between different amino acid nucleophilic group and HNE calculated at the SMD/MP2/6$311++G(d, p) / / B 3 L Y P / 6-31 G(d)$ level of theory in acetonitrile (ACN setup). resulting in zwitterionic intermediate $\mathbf{2}_{\mathbf{M}}$ via transition state $\mathbf{T S}_{1}$, similar to Lys amino acid. However, this step is not operative in all cases, and $\mathbf{2}_{\mathrm{M}}$ only forms when Arg attacks HNE with its $=\mathrm{NH}$ group (Figure 6 ) with free energy barrier of $20.9 \mathrm{kcal} \mathrm{mol}^{-1}$ (Table 1). In the next step, the thermodynamically relatively unstable $2_{\mathrm{M}}$ (less stable than reactants by $10.2 \mathrm{kcal} \mathrm{mol}^{-1}$ ) is transformed to the $3_{\mathrm{M}}$ via proton transfer which costs $27.5 \mathrm{kcal} \mathrm{mol}^{-1}$ in the case of reaction of $\mathrm{Arg}$ and its $=\mathrm{NH}$ group. As indicated earlier, in the cases of reaction of $\mathrm{Arg}-\mathrm{NH}_{2}$ group, $\mathrm{Cys}-\mathrm{SH}$ group and $\mathrm{His}-\mathrm{NH}$ group, the formation of Michael adduct $\mathbf{3}_{\mathrm{M}}$ occurs in a single step via transition state $\mathbf{T S}_{\mathbf{2 M}}$ without the formation of the unstable zwitterionic intermediate and requires 44.0, 55.7 and $59.6 \mathrm{kcal} \mathrm{mol}^{-1}$, for reaction of $\mathrm{Arg}-\mathrm{NH}_{2}$ group, $\mathrm{Cys}-\mathrm{SH}$ group and His $-\mathrm{NH}$ group with $\mathrm{HNE}$, respectively (Table 1). The Michael adducts are thermodynamically more stable than the starting reactants by $3.3,3.6$, 12.6 and $11.1 \mathrm{kcal} \mathrm{mol}^{-1}$, for adducts formed from $\mathrm{Arg}-\mathrm{NH}_{2}$ group, $\mathrm{Arg}=\mathrm{NH}$ group, $\mathrm{Cys}-\mathrm{SH}$ group and $\mathrm{His}-\mathrm{NH}$ group, respectively (Table 1 ). As a final step of the reaction, a hemiacetal adduct $\mathbf{4}$ is formed via additional proton transfer reaction with almost identical free energy barriers for all systems of $37.7 \mathrm{kcal} \mathrm{mol}^{-1}, 37.1 \mathrm{kcal} \mathrm{mol}^{-1}, 36.4 \mathrm{kcal} \mathrm{mol}^{-1}$ 
and $37.3 \mathrm{kcal} \mathrm{mol}{ }^{-1}$, for adducts formed from $\mathrm{Arg}-\mathrm{NH}_{2}$ group, $\mathrm{Arg}=\mathrm{NH}$ group, $\mathrm{Cys}-\mathrm{SH}$ group and $\mathrm{His}-\mathrm{NH}$ group, respectively (Table 1 ). Final hemiacetal adducts are thermodynamically more stable than reactants by 3.8, 5.3, 14.0 and $11.6 \mathrm{kcal} \mathrm{mol}{ }^{-1}$, for adducts formed from $\mathrm{Arg}-\mathrm{NH}_{2}$, Arg $=\mathrm{NH}, \mathrm{Cys}-\mathrm{SH}$, and $\mathrm{His}-\mathrm{NH}$ groups, respectively. Interestingly, Cys hemiacetal adduct is the most stable one, followed by His hemiacetal adduct, whereas both Arg adducts are relatively less stabilized when compared to Michael adducts and reactants. We should note that reactions in neat acetonitrile require very high free energy barriers (more than $35 \mathrm{kcal} \mathrm{mol}^{-1}$ ) indicating that these reactions are not feasible in physiological conditions.

However, it has been shown earlier that proton transfer reactions can be strongly catalyzed by water, where free energy barriers can significantly decrease. ${ }^{[20,28]}$ In order to check for the water catalysis effect, we added an explicit water molecule to the system $(\mathrm{ACN}+\mathrm{W})$. As we expect, free energy barriers for reactions involving proton transfer significantly decrease (Table 1, Figure 7). Interestingly, in contrast to ACN setup, zwitterion intermediate $\mathbf{2}_{\mathrm{M}}$ is now formed also in the reaction of $\mathrm{Arg}$ with its $-\mathrm{NH}_{2}$ group, whereas in the cases of Cys and His the zwitterionic intermediate is not located. Overall energetics is qualitatively similar to the ACN setup, with significantly lowered free energy barriers for proton transfer reactions via $\mathbf{T S}_{2 M}$ and $\mathbf{T S}_{3 \mathbf{M}}$ transition states. For example, in the conversion of zwitterionic intermediate $2_{M}$ to Michael adduct $\mathbf{3}_{M}$ in the case of adduct formed from $\mathrm{Arg}$ $-\mathrm{NH}$ group, free energy barrier decreases from 37.7 to $22.3 \mathrm{kcal} \mathrm{mol}^{-1}$. Still, the calculated free energy barriers are quite high, especially in the cases of Cys and His, but are significantly decreased compared to the ACN setup. Further addition of water molecules would probably decrease the

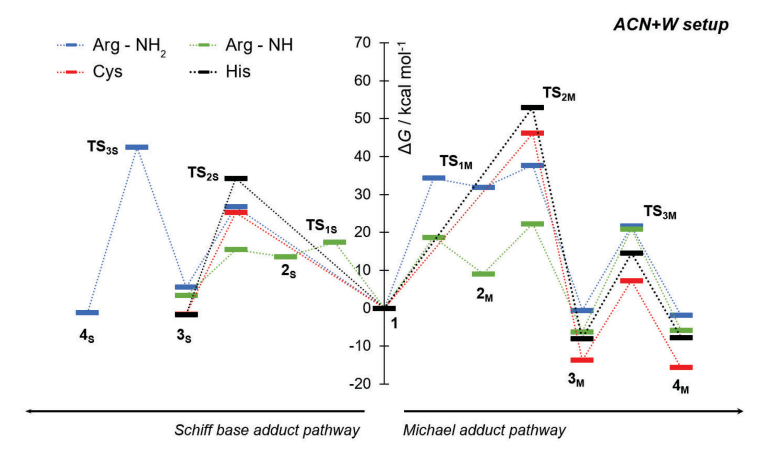

Figure 7. Free energy diagram for Michael adduct pathway (to the right) and Schiff base adduct pathway (to the left) for reaction between different amino acid nucleophilic group and HNE calculated at the SMD/MP2/6$311++G(d, p) / / B 3 L Y P / 6-31 G(d)$ level of theory in acetonitrile and one explicit water ( $\mathrm{ACN}+\mathrm{W}$ setup). barrier even more, as previously shown in the literature. ${ }^{[28]}$ The stability of the final Michael adducts and hemiacetals shows a similar trend as in the ACN setup, where Arg adducts are less stable than Cys and His adducts.

As stated in the previous paragraphs, the Michael addition adduct pathway is not the only one responsible for adduct formation upon reaction with RAs. In particular, instead of a nucleophilic attack to the $\mathrm{C}=\mathrm{C}$ double bond in $\mathrm{HNE}$, nucleophiles can also react with the carbonyl group, resulting in the formation of carbinolamine (or hemithioacetal in the case of Cys) $\mathbf{3}_{\mathrm{s}}$ and Schiff base adducts $\mathbf{4}_{\mathrm{s}}$ (Figure 6). In the ACN setup, all amino acids react directly via proton transfer yielding carbinolamine (or hemithioacetal in the case of Cys) adduct $\mathbf{3}_{\mathbf{s}}$ via transition state $\mathbf{T S} \mathbf{S}_{\mathbf{2}}$, with free energy barriers of 43.6, 33.7, 40.3 and $43.7 \mathrm{kcal}$ $\mathrm{mol}^{-1}$ for adducts formed from $\mathrm{Arg}-\mathrm{NH}_{2}$ group, Arg $=\mathrm{NH}$ group, Cys $-\mathrm{SH}$ group and $\mathrm{His}-\mathrm{NH}$ group, respectively (Table 1). Carbinolamine (or hemithioacetal in the case of Cys) adducts formed from $\mathrm{Arg}-\mathrm{NH}_{2}$ group, $\mathrm{Arg}=\mathrm{NH}$ group and Cys $-\mathrm{SH}$ group are by $5.2 \mathrm{kcal} \mathrm{mol}^{-1}, 2.6 \mathrm{kcal} \mathrm{mol}^{-1}$ and $0.3 \mathrm{kcal} \mathrm{mol}^{-1}$ thermodynamically less stable than the reactants, whereas His adduct is slightly stabilized by 0.6

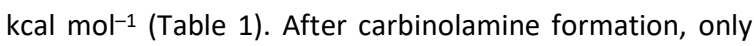
the adduct formed from $\mathrm{Arg}-\mathrm{NH}_{2}$ group reacts further via additional proton transfer and transition state $\mathbf{T S}_{3 \mathrm{~S}}$ with a large free energy barrier of $49.6 \mathrm{kcal} \mathrm{mol}^{-1}$ forming the final Schiff base adduct $4 \mathbf{s}$ which is thermo-dynamically less stable than reactants by $3.9 \mathrm{kcal} \mathrm{mol}^{-1}$ and thus unlikely to form.

We also studied the effect of the additional water added to the system ( $\mathrm{ACN}+\mathrm{W}$ setup). Just like in the Michael addition pathway, proton transfer reactions are catalyzed by ca. $15 \mathrm{kcal} \mathrm{mol}^{-1}$ (Table 1). Interestingly, in contrast to the ACN setup, a zwitterionic intermediate $\mathbf{2}_{s}$ is formed via transition state $\mathbf{T S}_{1 \mathrm{~s}}$ in the case of the reaction of $\mathrm{Arg}=\mathrm{NH}$ group with HNE. All other reaction steps are identical to the mechanism obtained within the ACN setup, but the final Schiff base adduct $4 \mathrm{~s}$ in the case of $\mathrm{Arg}-\mathrm{NH}_{2}$ group is thermodynamically more stable than the starting reactants by $1.2 \mathrm{kcal} \mathrm{mol}^{-1}$.

\section{Modification of Amino Acids with ONE}

In this Section, we will describe the reactivity of Arg, Cys and His amino acids with ONE, which has a keto group instead of a hydroxyl group in comparison to HNE (Figure 1). According to our calculations, all studied amino acids react in a manner similar to $\mathrm{HNE}$, yielding similar products. However, the reaction mechanism is not identical and some of the reactions are not possible, in particular the cyclization of Michael adducts to hemiacetals.

Firstly, we will focus on the reaction of Arg amino acid with ONE. Nucleophilic addition of Arg to ONE can occur in two ways, depending whether $-\mathrm{NH}_{2}$ group (Figure 8a) or 
$=\mathrm{NH}$ group (Figure $8 \mathrm{~b}$ ), undergoes the nucleophilic addition to the ONE C=C double bond. This results in the formation of Michael adduct as the final product of the reaction since further cyclization to hemiacetals is not possible due to the lack of the nucleophilic hydroxyl group present in HNE. Similar to the Schiff base adduct pathway in reactions of Arg with HNE, two possible reaction routes exist, depending which nucleophilic site of Arg reacts with the carbonyl group of ONE (Figure 9).

If $\operatorname{Arg}-\mathrm{NH}_{2}$ group reacts with ONE, both carbinolamine and Schiff base adduct are possible reaction products (Figure 9a). On the other hand, if $\mathrm{Arg}=\mathrm{NH}$ group reacts with ONE, only carbinolamine adduct is formed (Figure 9b). This mechanism is identical to the one observed in reactions of Arg with HNE.

As a next example, we will study the nucleophilic attack of the Cys -SH group to ONE. In this case, the Michael addition pathway yields only Michael adduct (Figure 10a) while Schiff base pathway results in hemithioacetal adduct as the final product of the reaction (Figure 10b).

Finally, we show a reaction mechanism for reaction of ONE with the - $\mathrm{NH}$ group in imidazole His side chain ring, resulting in Michael adduct (Figure 11a) and carbinolamine adduct (Figure 11b), similar to the reaction of Cys with ONE.

Relative free energies, calculated at the SMD/MP2/ $6-311++G(d, p) / / B 3 L Y P / 6-31 G(d)$ level of theory in two different setups, $A C N$ and $A C N+W$ (see Computational Details) for the reaction between amino acids and ONE, is given in the Table 2. Free energy diagram showing formation of described adducts for all amino acids upon

\section{Michael adduct pathway}

a)

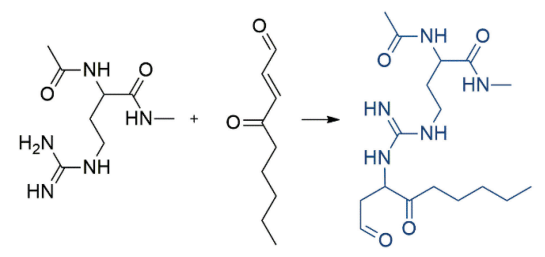

b)

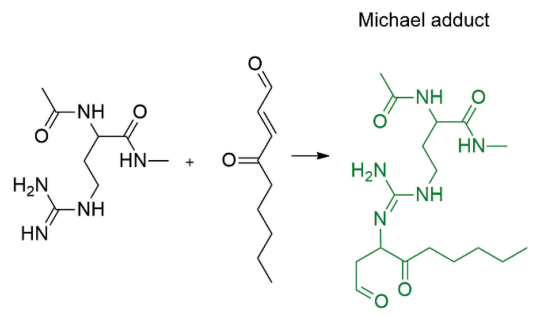

Michael adduct

Figure 8. Michael adduct pathway for reaction between $-\mathrm{NH}_{2}$ group of arginine (panel a) and $=\mathrm{NH}$ group of arginine (panel b) with ONE.

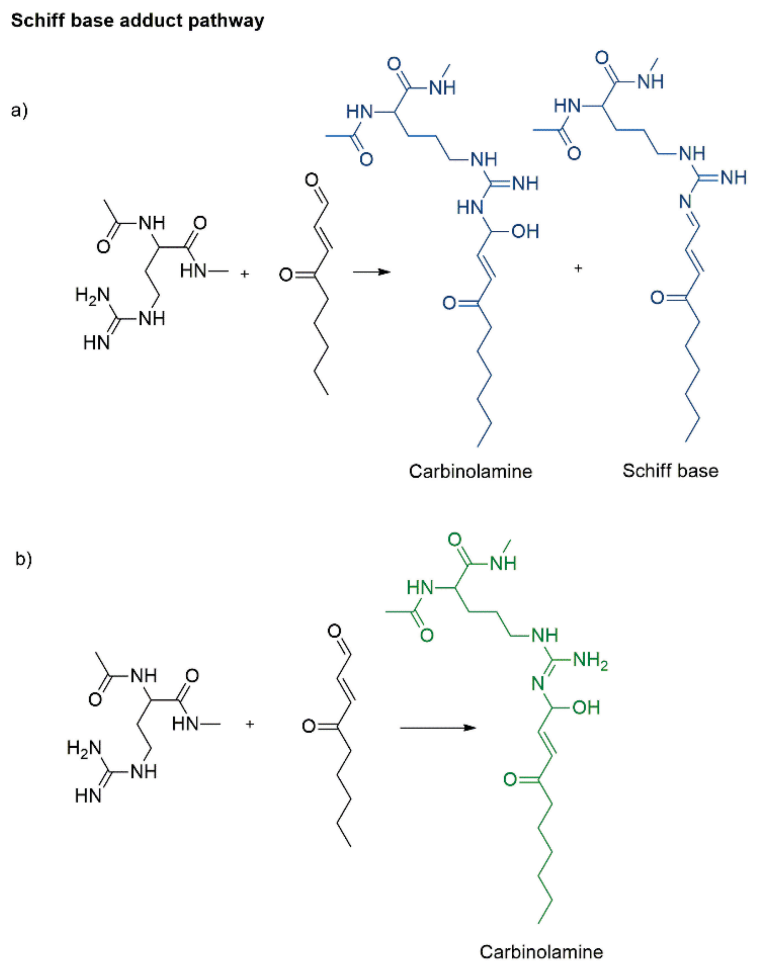

Figure 9. Schiff base adduct pathway for reaction between $-\mathrm{NH}_{2}$ group of arginine (panel a) and $=\mathrm{NH}$ group of arginine (panel b) with ONE.

Michael adduct pathway

a)<smiles>CCCCCC(=O)C(CC=O)CSC(CS)C(=O)NC(C)=O</smiles>

Michael adduct

Schiff base adduct pathway

b)

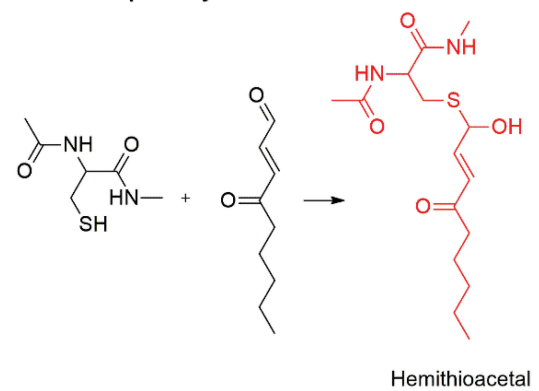

Figure 10. Michael adduct pathway (panel a) and Schiff base adduct pathway (panel b) for reaction between $-\mathrm{SH}$ group of cysteine and ONE. 


\section{Michael adduct pathway}

a)<smiles>CCCCCC(=O)/C=C/C=O</smiles>

Michael adduct

Schiff base adduct pathway

b)

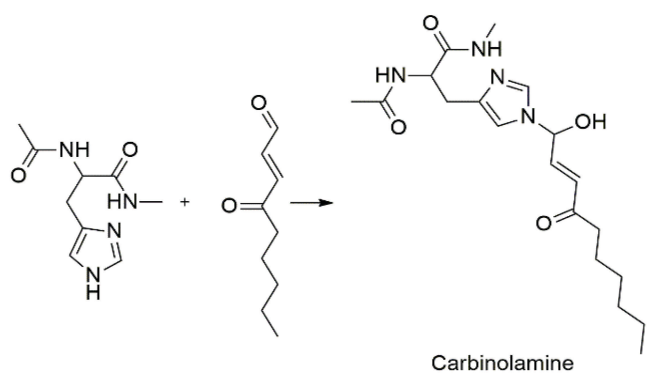

Figure 11. Michael adduct pathway (panel a) and Schiff base adduct pathway (panel b) for reaction between -NH group of histidine and ONE. reaction with $\mathrm{ONE}$ in acetonitrile $(\mathrm{ACN})$ is presented in Figure 12, whereas free energy diagram for the reaction mechanism with one explicit water in acetonitrile ( $\mathrm{ACN}+\mathrm{W})$ is presented in Figure 13.

Similar to the data analysis in the case of HNE, we will first focus on the mechanism of reaction along the Michael pathway in the ACN setup (Figure 12, Table 2). The formation of the zwitterionic intermediate $\mathbf{2}_{\mathbf{M}}$, via transition state $\mathbf{T S}_{1 \mathrm{M}}$, is observed for both Arg reactive

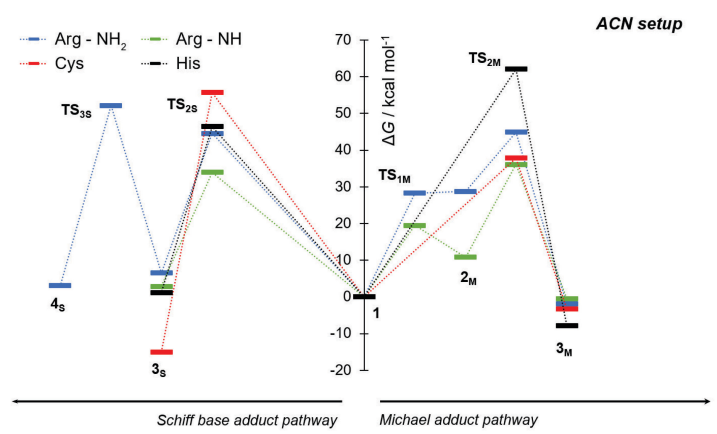

Figure 12. Free energy diagram for Michael adduct pathway (to the right) and Schiff base adduct pathway (to the left) for reaction between different amino acid nucleophilic group and ONE calculated at the SMD/MP2/6$311++G(d, p) / / B 3 L Y P / 6-31 G(d)$ level of theory in acetonitrile (ACN setup).

Table 2. Relative free energies studied compounds vs. reactants in $\mathrm{kcal} \mathrm{mol}^{-1}$ for the reaction between ONE and different amino acids along Michael adduct pathway (MA pathway) and Schiff base adduct pathway (SB pathway) calculated at the SMD/MP2/6$311++G(d, p) / / B 3 L Y P / 6-31 G(d)$ level of theory in acetonitrile (ACN setup) and acetonitrile with one explicit water molecule (ACN+W setup).

\begin{tabular}{|c|c|c|c|c|c|c|c|c|c|c|}
\hline \multirow{2}{*}{ ONE } & \multicolumn{5}{|c|}{ ACN setup } & \multicolumn{5}{|c|}{$\mathrm{ACN}+\mathrm{W}$ setup } \\
\hline & $\operatorname{Arg}-\mathrm{NH}_{2}$ & $\operatorname{Arg}=\mathrm{NH}$ & Cys $-\mathrm{SH}$ & $\mathrm{His}-\mathrm{NH}$ & Lys ${ }^{(a)}-\mathrm{NH}_{2}$ & $\operatorname{Arg}-\mathrm{NH}_{2}$ & $\mathrm{Arg}=\mathrm{NH}$ & Cys $-\mathrm{SH}$ & $\mathrm{His}-\mathrm{NH}$ & Lys ${ }^{(a)}-\mathrm{NH}_{2}$ \\
\hline \multicolumn{11}{|c|}{ MA-pathway } \\
\hline 1 & 0.0 & 0.0 & 0.0 & 0.0 & 0.0 & 0.0 & 0.0 & 0.0 & 0.0 & 0.0 \\
\hline $\mathrm{TS}_{1 \mathrm{M}}$ & - & 20.9 & - & - & 15.2 & 34.3 & 18.7 & - & - & 15.0 \\
\hline $2 \mathrm{M}$ & - & 10.2 & - & - & 8.2 & 31.9 & 9.1 & - & - & 6.3 \\
\hline $\mathrm{TS}_{2 \mathrm{M}}$ & 44.0 & 37.7 & 55.7 & 59.6 & 32.7 & 37.7 & 22.3 & 46.2 & 53.0 & 17.9 \\
\hline 3 & -3.3 & -3.6 & -12.6 & -11.1 & -7.1 & -0.6 & -6.2 & -13.7 & -8.0 & -10.5 \\
\hline \multicolumn{11}{|c|}{ SB-pathway } \\
\hline 1 & 0.0 & 0.0 & 0.0 & 0.0 & 0.0 & 0.0 & 0.0 & 0.0 & 0.0 & 0.0 \\
\hline $\mathrm{TS}_{1 \mathrm{~S}}$ & - & - & - & - & - & - & 17.4 & - & - & - \\
\hline $2 s$ & - & - & - & - & - & - & 13.6 & - & - & - \\
\hline $\mathrm{TS}_{2 \mathrm{~S}}$ & 43.6 & 33.7 & 40.3 & 43.7 & 27.9 & 26.8 & 15.5 & 25.3 & 34.2 & 10.0 \\
\hline $3 \mathrm{~s}$ & 5.2 & 2.6 & 0.3 & -0.6 & -0.8 & 5.6 & 3.4 & -1.5 & -1.7 & -2.9 \\
\hline $\mathrm{TS}_{3 \mathrm{~S}}$ & 54.8 & - & - & - & 39.3 & 42.5 & - & - & - & 22.9 \\
\hline 4 & 3.9 & - & - & - & -5.4 & -1.2 & - & - & - & -7.3 \\
\hline
\end{tabular}




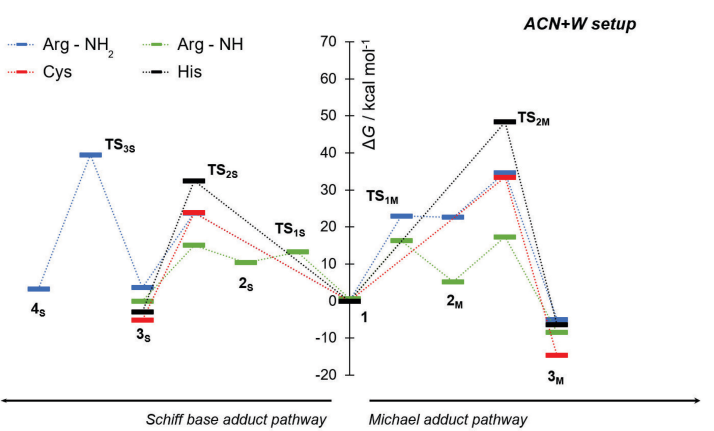

Figure 13. Free energy diagram for Michael adduct pathway (to the right) and Schiff base adduct pathway (to the left) for reaction between different amino acid nucleophilic group and ONE calculated at the SMD/MP2/6$311++G(d, p) / / B 3 L Y P / 6-31 G(d)$ level of theory in acetonitrile and one explicit water (ACN+W setup).

groups, in contrast to HNE where only =NH group reacts in this way. Free energy barriers for reaction of Arg with its $-\mathrm{NH}_{2}$ and $=\mathrm{NH}$ group amount to 28.3 and $19.5 \mathrm{kcal} \mathrm{mol}^{-1}$, respectively, resulting in unstable intermediates $\mathbf{2}_{\mathrm{M}}$. Interestingly, the zwitterionic intermediate formed when Arg $-\mathrm{NH}_{2}$ group reacts with ONE is very unstable (even slightly less stable than the $\mathbf{T S}_{1 \mathrm{M}}$ when thermal corrections are included), but we were able to locate it in contrast to the identical reaction with HNE. Both zwitterionic intermediates then undergo proton transfer reaction via transition state $\mathbf{T S}_{\mathbf{2 M}}$, resulting in the final Michael adducts $\mathbf{3}_{\mathrm{M}}$, which are by 1.9 and $0.5 \mathrm{kcal} \mathrm{mol}^{-1}$ thermodynamically more stable than the reactants. The formation of Michael adducts are kinetically prohibited in physiological conditions (at human body temperature of $37^{\circ} \mathrm{C}$ ) in neat acetonitrile since the free energy barriers involve transition states which are by $44.9 \mathrm{kcal} \mathrm{mol}^{-1}$ and $36.1 \mathrm{kcal} \mathrm{mol}^{-1}$ higher than the reactants. In the case of Cys and His amino acids, no zwitterionic intermediates $\mathbf{2}_{\mathrm{M}}$ are located and both amino acids directly undergo proton transfer reaction and carbon-carbon coupling resulting in Michael adducts $3_{\mathrm{M}}$ which are thermodynamically more stable than the reactants by 3.2 and $7.8 \mathrm{kcal} \mathrm{mol}^{-1}$, respectively. However, similar to the case of Arg amino acid, free energy barriers for formation of Michael adducts are again prohibitively high in neat acetonitrile, especially in the case of His amino acid where the barrier is larger than $60 \mathrm{kcal} \mathrm{mol}^{-1}$.

As previously shown in the case of amino acid reactions with $\mathrm{HNE}$, proton transfer reactions can be catalyzed by the addition of a water molecule as studied within the $\mathrm{ACN}+\mathrm{W}$ setup (see Computational Details). This is readily visible in this case as well, where prohibitively large free energy barriers are reduced by $10-15 \mathrm{kcal} \mathrm{mol}^{-1}$ (Table 2), resulting in more realistic barriers for proton transfer, especially for reaction of Arg amino acid with ONE. In addition, final Michael adducts are thermodynamically more stable than the reactants by $5.0,8.4,14.7$ and $6.4 \mathrm{kcal} \mathrm{mol}^{-1}$ for adducts formed from Arg $-\mathrm{NH}_{2}$ group, Arg =NH group, Cys $-\mathrm{SH}$ group and $\mathrm{His}-\mathrm{NH}$ group, respectively (Table 2 ).

In addition to the Michael adduct pathway, the Schiff base adduct pathway is operative in reaction of amino acids with ONE as well. The possible products of the reaction are identical to the case of ONE in ACN setup, resulting in the Schiff base adduct $\mathbf{4}_{\mathrm{s}}$ only for adducts formed from Arg - $\mathrm{NH}_{2}$ group, while all other amino acids finish in carbinolamine (or hemithioacetal in the case of Cys) adducts $\mathbf{3}_{\mathrm{s}}$. In particular, free energy barriers for formation of carbinolamine (or hemithioacetal in the case of Cys) via transition state $\mathbf{T S}_{2 \mathrm{~s}}$ in acetonitrile amount to $44.5,34.0$, 55.7 and $46.4 \mathrm{kcal} \mathrm{mol}^{-1}$ for adducts formed from $\mathrm{Arg}-\mathrm{NH}_{2}$ group, $\mathrm{Arg}=\mathrm{NH}$ group, $\mathrm{Cys}-\mathrm{SH}$ group and His $-\mathrm{NH}$ group, respectively (Table 2 ). Carbinolamine adducts $\mathbf{3}_{\boldsymbol{s}}$ are thermodynamically less stable than the reactants, expect in the case of Cys adduct where the hemithioacetal adduct in the case of adduct is by $15.0 \mathrm{kcal} \mathrm{mol}^{-1}$ more stable than the starting reactants. Like in the case of HNE, thermodynamically unstable Schiff base adduct $4 \mathrm{~s}$, less stable than the reactants by $3.0 \mathrm{kcal} \mathrm{mol}^{-1}$, is formed only from the carbinolamine adduct formed from $\operatorname{Arg}-\mathrm{NH}_{2}$ group via transition state $\mathbf{T S}_{3 \mathrm{~S}}$ with prohibitively large free energy barrier of $45.7 \mathrm{kcal} \mathrm{mol}^{-1}$.

The strong catalytic effect of water is visible in the Schiff base adduct pathway as well, where addition of water molecule within the $\mathrm{ACN}+\mathrm{W}$ setup results in decrease of free energy barriers for proton transfer reactions by $15-20$ $\mathrm{kcal} \mathrm{mol}^{-1}$. In addition, the zwitterionic intermediate $\mathbf{2} \mathrm{s}$ is formed via transition state $\mathbf{T S}_{1 \mathrm{~s}}$ in the case of the reaction of $\mathrm{Arg}=\mathrm{NH}$ group with $\mathrm{ONE}$, similar to the situation observed in identical reaction with HNE. Carbinolamine adducts (or hemithioacetal in the case of Cys) $\mathbf{3}_{\mathbf{s}}$ are thermodynamically less stable than reactants in the case of Arg adducts (by 3.7 and $0.7 \mathrm{kcal} \mathrm{mol}^{-1}$ ), whereas in the case of Cys and His, final carbinolamine adducts (or hemithioacetal in the case of (ys) are more stable than the reactants by 5.2 and $3.0 \mathrm{kcal} \mathrm{mol}^{-1}$. Finally, Schiff base adduct $\mathbf{4}_{\mathrm{s}}$ is formed only from the carbinolamine adduct formed from Arg $-\mathrm{NH}_{2}$ group via transition state $\mathrm{TS}_{3 \text { s }}$ with free energy barrier of $35.8 \mathrm{kcal} \mathrm{mol}^{-1}$, which is by ca. $10 \mathrm{kcal} \mathrm{mol}^{-1}$ lower than in the case of ACN setup without explicit water.

\section{DISCUSSION AND CONCLUSION}

In this work, we described rich chemical transformations in the reactions between reactive aldehydes and Arg, Cys and His amino acids. The reaction mechanism presented here is quite complex, and results in a number of different products along the reaction route. However, the reaction mechanism of covalent modification of studied amino acids 
with HNE and ONE, although similar to the mechanism of lysine studied in our previous work, ${ }^{[20]}$ is a bit different. In particular, free energy barriers for reactions of Arg, Cys and His with HNE for both Michael addition pathway and Schiff base adduct pathway are in general higher for all studied reaction steps. Specifically, the rate determining step involving transition state $\mathbf{T S}_{\mathbf{2}} \mathbf{M}$ in the case of Cys and His and neat acetonitrile (ACN setup) involves free energy barriers which are very high, being over $50 \mathrm{kcal} \mathrm{mol}^{-1}$, whereas in the case of $\mathrm{Arg}$, the analogous free energy barriers for reaction of Arg with HNE are lower and comparable with Lys (Table 1). In the case of the Schiff base adduct pathway in acetonitrile, free energy barriers which determine the rate of the reaction are lower in comparison to Michael adduct pathway, but are still over $30 \mathrm{kcal} \mathrm{mol}^{-1}$. Upon addition of water (in the $\mathrm{ACN}+\mathrm{W}$ setup), the situation changes a little and barriers are significantly lowered, but are still quite high as compared to Lys for both pathways.

The calculated energy profiles and suggest that Lys should be the most reactive amino acid in the series for reaction with $\mathrm{HNE}$, followed by $\mathrm{Arg}$, Cys and His. This is in apparent contrast to LC-MS experiments where Cys amino acid should be the most reactive amino acid in water. ${ }^{[16,29]}$ However, the analysis of the reaction mechanism studied here is performed in acetonitrile and acetonitrile/water mixtures, whereas the LC-MS experiments were performed in phosphate buffers. ${ }^{[17]}$ This actually poses a problem, since HNE and ONE are very sparsely soluble in water and described experiments in water have been performed in 20x excess of RAs vs peptide. Therefore, the experimental conditions were not identical to our setup, which may partly explain the observed differences in reaction kinetics. In addition to low solubility of RAs in water, amino acids have different protonation state in water too (depending on their $\mathrm{p} K_{\mathrm{a}}$ value) in contrast to acetonitrile. In order to generate a nucleophilic specie ready for nucleophilic addition (see Results section and individual reaction mechanisms for single amino acids), one has to take into account also the deprotonation energy of amino acid in water which is higher for Arg than for Lys, Cys and $\mathrm{His}$ (corresponding $\mathrm{p} K_{\mathrm{a}}$ values are $12.5,10.8,8.3$ and 6.0 , respectively). Therefore, the reactivity order might significantly change in water, depending on the amino acid deprotonation energy. The reason why we chose acetonitrile instead of water is motivated by the fact that HNE and ONE have very low solubility in water and it is difficult to control the reaction without knowing exactly how much of the reactive aldehyde actually enters the reaction. This in turn can also lead to the reactions with a different stoichiometry than predicted, thus even further blurring the details of the reaction mechanism.
In the case of the reaction of studied amino acids with $\mathrm{ONE}$, only adducts formed from Arg =NH group have barriers which are relatively small (below $25 \mathrm{kcal}$ $\mathrm{mol}^{-1}$ ) and can be relevant to the physiological conditions, although free energy barriers for other systems are lower when compared to HNE, indicating that ONE might be more reactive than HNE as experimentally suggested. Still, the free energy reaction barriers are still quite high for His and Cys, suggesting that they are less reactive than Arg in contrast to LC-MS experimental measurements in water by Petersen. Similar to the case with $\mathrm{HNE}$, addition of water has a strong effect on the reduction of free energy barriers involving proton transfer (Table 2). Here, we should mention that reaction between Lys and ONE was not studied in this work. Surprisingly, for this particular reaction Sayre and coworkers have proposed that the main reaction product of Lys addition to ONE via Michael adduct pathway is not the Michael adduct. Instead, the ketoamine adduct is formed in contrast to other amino acids which produce Michael adducts. ${ }^{[14]}$ The details of the reaction mechanism between Lys and ONE are not understood well and are currently under investigation in our group.

In summary, we presented in this work the intricate details of the reaction mechanism of modification of Arg, Cys and His amino acids with reactive aldehydes HNE and ONE in acetonitrile and acetonitrile/water systems. We identified all of the reaction steps and calculated complete energetics of the reactions. We found out that in the Michael addition pathway with both HNE and ONE, $\mathrm{Arg}$ has the lowest reaction barriers, whereas Cys and His have prohibitively large barriers, even with the addition of water which significantly lowers high free energy barriers in proton transfer reactions for all systems. In the case of Schiff base adduct pathway, the situation is similar and Arg has the lowest free energy barriers along the reaction pathway in contrast to His and Cys. These results are in contradiction with available experimental results where it has been found that Cys is more reactive than other amino acids, ${ }^{[16,17]}$ but we should stress that these experiments have been performed in water in contrast to the present study which used acetonitrile and acetonitrile/water as model solvents. Still, the reaction mechanism should be similar in water as well, although additional facts, such as low solubility of RAs and deprotonation energy should be taken into account. Finally, the free energy barriers involving His and Cys amino acids are relatively smaller than in the case of the reaction with $\mathrm{HNE}$, suggesting that $\mathrm{ONE}$ is more reactive than HNE as experimentally suggested in water solutions. ${ }^{[17]}$ 
Acknowledgment. This study was supported by Croatian Science Foundation, project no. UIP-2014-09-6090. The calculations were performed using the resources of computer cluster Isabella based in SRCE - University of Zagreb University Computing Center. We also thank Dr. Katarina Vazdar for valuable comments and critical reading of the manuscript.

\section{REFERENCES}

[1] H. Esterbauer, R. J. Schaur, H. Zollner, Free Radic. Biol. Med. 1991, 11, 81-128.

https://doi.org/10.1016/0891-5849(91)90192-6

[2] L. Seon Hwa, T. Oe, I. A. Blair, Science 2001, 292, 2083-2086.

https://doi.org/10.1126/science.1059501

[3] A. Catala, Chem. Phys. Lipids 2009, 157, 1-11. https://doi.org/10.1016/j.chemphyslip.2008.09.004

[4] K. Vazdar, D. Vojta, D. Margetić, M. Vazdar, Chem. Res. Toxicol. 2017, 30, 840-850.

https://doi.org/10.1021/acs.chemrestox.6b00443

[5] G. Poli, R. J. Schaur, W. G. Siems, G. Leonarduzzi, Med. Res. Rev. 2008, 28, 569-631.

https://doi.org/10.1002/med.20117

[6] R. J. Schaur, Mol. Aspects Med. 2003, 24, 149-159. https://doi.org/10.1016/S0098-2997(03)00009-8

[7] A. L. Isom, S. Barnes, L. Wilson, M. Kirk, L. Coward, V. Darley-Usmar, J. Am. Soc. Mass Spectrom. 2004, 15, 1136-1147.

https://doi.org/10.1016/j.jasms.2004.03.013

[8] W. Siems, T. Grune, Mol. Aspects Med. 2003, 24, 167-175.

https://doi.org/10.1016/S0098-2997(03)00011-6

[9] T. Grune, K. J. A. Davies, Mol. Aspects Med. 2003, 24, 195-204.

https://doi.org/10.1016/S0098-2997(03)00014-1

[10] G. Barrera, S. Pizzimenti, E. S. Ciamporcero, M. Daga, C. Ullio, A. Arcaro, G. P. Cetrangolo, C. Ferretti, C. Dianzani, A. Lepore, et al., Antioxid. Redox Signal. 2014, 22, 1681-1702.

https://doi.org/10.1089/ars.2014.6166

[11] M. Csala, T. Kardon, B. Legeza, B. Lizák, J. Mandl, É. Margittai, F. Puskás, P. Száraz, P. Szelényi, G. Bánhegyi, Biochim. Biophys. Acta - Mol. Basis Dis. 2015, 1852, 826-838. https://doi.org/10.1016/j.bbadis.2015.01.015

[12] N. Žarković, A. Čipak, M. Jaganjac, S. Borović, K. Žarković, J. Proteomics 2013, 92, 239-247. https://doi.org/10.1016/j.jprot.2013.02.004
[13] L. M. Sayre, P. K. Arora, R. S. Iyer, R. G. Salomon, Chem. Res. Toxicol. 1993, 6, 19-22. https://doi.org/10.1021/tx00031a002

[14] L. M. Sayre, D. Lin, Q. Yuan, X. Zhu, X. Tang, Drug Metab. Rev. 2006, 38, 651-675. https://doi.org/10.1080/03602530600959508

[15] D. V Nadkarni, L. M. Sayre, Chem. Res. Toxicol 1996, 8, 284-291. https://doi.org/10.1021/tx00044a014

[16] D. Lin, H. G. Lee, Q. Liu, G. Perry, M. A. Smith, L. M. Sayre, Chem. Res. Toxicol. 2005, 18, 1219-1231. https://doi.org/10.1021/tx050080q

[17] J. A. Doorn, D. R. Petersen, Chem. Res. Toxicol. 2002, 15, 1445-1450. https://doi.org/10.1021/tx025590o

[18] J. P. Castro, T. Jung, T. Grune, W. Siems, Free Radic. Biol. Med. 2017, 111, 309-315.

https://doi.org/10.1016/j.freeradbiomed.2016.10.497

[19] M. Carini, G. Aldini, R. M. Facino, Mass Spectrom. Rev. 2004, 23, 281-305. https://doi.org/10.1002/mas.10076

[20] S. Škulj, K. Vazdar, D. Margetić, M. Vazdar, J. Org. Chem. 2019, 84, 526-535. https://doi.org/10.1021/acs.joc.8b02231

[21] A. V Marenich, C. J. Cramer, D. G. Truhlar, J. Phys. Chem. B 2009, 113, 6378-6396. https://doi.org/10.1021/jp810292n

[22] J. Tomasi, B. Mennucci, R. Cammi, Chem. Rev. 2005, 105, 2999-3094. https://doi.org/10.1021/cr9904009

[23] A. D. Becke, J. Chem. Phys. 1993, 98, 5648-5652. https://doi.org/10.1063/1.464913

[24] P. J. Stephens, F. J. Devlin, C. F. Chabalowski, M. J. Frisch, J. Phys. Chem. 1994, 98, 11623-11627. https://doi.org/10.1021/j100096a001

[25] W. J. Hehre, K. Ditchfield, J. A. Pople, J. Chem. Phys. 1972, 56, 2257-2261. https://doi.org/10.1063/1.1677527

[26] P. C. Hariharan, J. A. Pople, Theor. Chim. Acta 1973, 28, 213-222. https://doi.org/10.1007/BF00533485

[27] M. J. Frisch, G. W. Trucks, H. B. Schlegel, G. E. Scuseria, M. A. Robb, J. R. Cheeseman, G. Scalmani, V. Barone, B. Mennucci, G. A. Petersson, et al., Gaussian, Inc., Wallingford CT, 2009.

[28] Y. Ding, Y. Cui, T. Li, J. Phys. Chem. A 2015, 119, 4252-4260.

https://doi.org/10.1021/acs.jpca.5b02186

[29] D. R. Petersen, J. A. Doorn, Free Radic. Biol. Med. 2004, 37, 937-945.

https://doi.org/10.1016/j.freeradbiomed.2004.06.012 